\title{
A Machine Learning Potential for Hexagonal Boron Nitride Applied to Thermally and Mechanically Induced Rippling
}

\author{
Fabian L. Thiemann, ${ }^{\dagger} \ddagger$ Patrick Rowe, ${ }^{\dagger}$ Erich A. Müller, ${ }^{\ddagger}$ and Angelos \\ Michaelides*, $\dagger$ \\ $\dagger$ Thomas Young Centre, London Centre for Nanotechnology, and Department of Physics \\ and Astronomy, University College London, Gower Street, London, WC1E 6BT, United \\ Kingdom \\ $\ddagger$ Department of Chemical Engineering, Imperial College London, South Kensington \\ Campus, London SW7 2AZ, United Kingdom \\ E-mail: angelos.michaelides@ucl.ac.uk
}

\section{Abstract}

We introduce an interatomic potential for hexagonal boron nitride (hBN) based on the Gaussian approximation potential (GAP) machine learning methodology. The potential is based on a training set of configurations collected from density functional theory (DFT) simulations and is capable of treating bulk and multilayer hBN as well as nanotubes of arbitrary chirality. The developed force field faithfully reproduces the potential energy surface predicted by DFT while improving the efficiency by several orders of magnitude. We test our potential by comparing formation energies, geometrical properties, phonon dispersion spectra and mechanical properties with respect to benchmark DFT calculations and experiments. In addition, we use our model and a recently developed graphene-GAP to analyse and compare thermally and mechanically induced rippling in large scale two-dimensional (2D) hBN and graphene. Both materials show almost identical scaling behaviour with an exponent of $\eta \approx 0.85$ for the height fluctuations agreeing well with the theory of flexible membranes. Based on its lower resistance to bending, however, hBN experiences slightly larger out-of-plane deviations both at zero and finite applied external strain. Upon compression a phase transition from incoherent ripple motion to soliton-ripples is observed for both materials. Our potential is freely available online at [http://www.libatoms.org].

\section{Introduction}

Hexagonal boron nitride (hBN), though less well-known than its isostructural analogue, graphene, is no less fascinating from the perspective of its properties and potential applications. The insulating character, ${ }^{1}$ resistance to oxidation, ${ }^{2}$ high thermal conductivity, ${ }^{3}$ and mechanical strength ${ }^{4}$ of hBN have been exploited in various fields ranging from electronic devices, ${ }^{5,6}$ water purification, ${ }^{7}$ and industrial chemistry $^{8-11}$ to biomedical science and engineering. ${ }^{12-14}$ Yet, certain aspects of the behaviour of low-dimensional hBN remain to be rationalised and explored further. For instance, the origin of experimentally observed differences in friction ${ }^{15}$ and surface charge ${ }^{16,17}$ between graphene and hBN surfaces in contact with water requires a deeper understanding on the microscopic level ${ }^{18}$ to exploit the full potential of hBN for desalination processes and 
osmotic power generation. ${ }^{19}$ Similarly, the impact of intrinsic ripples on layered $\mathrm{hBN}$ has not received as much attention as for graphene, ${ }^{20,21}$ where these out-of-plane deviations have a significant impact on properties such as graphene's bandgap ${ }^{22-24}$ or reactivity. ${ }^{25}$ Assuming analogous behaviour for two-dimensional (2D) hBN the ability to predict, and potentially adjust the ripple texture through strain would be of value to future applications. ${ }^{26}$

Molecular dynamics (MD) simulations offer a computational approach to gain insight into nanoscale properties of materials. A faithful representation of the potential energy surface (PES) is a crucial requirement for reliable simulations, in particular an accurate description of the phonon dispersion spectrum which determines a variety of mechanical and thermodynamic properties as well as diffusion processes across low-dimensional materials. Due to the strong coupling between the adsorbate's motion and the phonon modes of the solid, ${ }^{27-30}$ potential inaccuracies in the predicted lattice vibrations are directly propagated to dynamical and interfacial properties. Various force fields for $\mathrm{hBN}$ are available, including Tersoff-type potentials, ${ }^{31-41}$ reactive force fields, ${ }^{42-46}$ and potentials fitted to density functional theory (DFT). ${ }^{47}$ Despite the valuable and excellent work on thermal and mechanical properties of hBN allotropes ${ }^{48-51}$ based on these established models and notwithstanding their computational efficiency, none of them predicts the vibrational properties in good agreement with experiments as we learned in the course of this work. Thus, these models cannot be expected to give reliable results for our systems of interest.

Incorporating electronic structure based methods and performing $a b$ initio molecular dynamics (AIMD) represents an alternative approach which often offers a significantly more accurate description of the PES and the phonon dispersion spectrum. DFT driven AIMD has been employed to study hBN in various contexts including proton transfer through a hBN sheet, ${ }^{52}$ interfacial behaviour with water, ${ }^{53}$ and the impact of strain on the vibrational properties of single and multiple layers of hBN. ${ }^{54}$ These methods, however, come with a high computational cost that severely restricts the accessible system sizes and time scales. Finitesize effects remain a practical issue of AIMD simulations and can compromise the reliability of calculated transport properties for confined fluids. ${ }^{55}$ This is also true for ripples where the amplitude of the out-of-plane deviations is partially determined by the dimensions of the $2 \mathrm{D}$ layer. ${ }^{21,56}$

The rise of machine learning (ML) methodologies and their fruitful application in the development of interatomic potentials has provided a pathway for achieving an accuracy close to that of their $a b$ initio reference while lowering the computational costs by several orders of magnitude ${ }^{57-63}$. These potentials are based on a transformation of atomic coordinates using high dimensional descriptors ${ }^{58}$ which serve as input for the ML algorithm to establish a structure-energy mapping. In order to actually "learn" this relationship, a large database of configurations and related $a b$ initio energies and forces is required. In recent years, many ML-based potentials have been developed for a significant number of materials spanning a variety of algorithms including Gaussian kernel regression, artificial neural networks and permutationally invariant polynomials. ${ }^{64-68} \mathrm{~A}$ machine learning potential based on the Gaussian Approximation Potential (GAP) ${ }^{65,69}$ framework was recently presented by some of us for hBN's isostructural analogue, graphene. ${ }^{70}$ This model achieves a very good agreement with experiments throughout a large range of properties including the thermal expansion and phonon dispersion spectrum. GAP models also have been proposed to study amorphous and crystalline carbon, ${ }^{71,72}$ tungsten, ${ }^{73}$ silicon, ${ }^{65}$ as well as hybrid perovskites. ${ }^{74}$

In this work we introduce a GAP for $h B N$ which is able to treat the bulk phase, isolated and multilayered sheets of hBN, as well as nanotubes of arbitrary chirality. We evaluate the performance of our model by comparing against 
DFT as well as frequently applied force fields for a variety of properties. In particular, significant improvements for the phonon spectra are obtained with the hBN-GAP compared to established force fields. We, then, perform largescale MD simulations using our new potential and the recently published graphene-GAP 70 to investigate the behaviour of thermally and mechanically induced rippling in $2 \mathrm{D} \mathrm{hBN}$ and graphene. We find an almost identical scaling exponent for both materials, while the amplitude and shape of the height fluctuations depends on the material's properties as well as on the applied strain. Looking forward, the hBN-GAP will be particularly valuable where an accurate description of the system's vibrations and dynamics is required and where the desired length and timescales are not accessible with electronic structure methods. This includes quantitative studies on diffusion of adsorbates across layered hBN or through hBN nanotubes.

The remainder of this paper is structured as follows. Next, we give a brief overview of the GAP methodology as well as a detailed explanation of technical aspects which are crucial to consider in the construction of a ML potential. This is followed by a detailed evaluation of the accuracy of the hBN-GAP. The results of the rippling analysis are reported before we draw the conclusions of this study.

\section{Theory and Computational Details}

\section{Construction of the hBN-GAP}

A detailed derivation of the theory behind the GAP framework is available elsewhere. ${ }^{65,69-71,73}$ For completeness, we will give an abridged explanation and focus on the practical issues within the construction. GAP mimics the Born-Oppenheimer PES without treating electrons explicitly by establishing a link between structure and energy based on a transformation of the atomic positions into local environments using so-called descriptors. ${ }^{65}$ To this end, the target PES is represented by a large database of $a b$ initio energies which is then interpolated by using Gaussian kernel regression (GKR). The decomposition into local contributions is an approximation inherent to all interatomic potentials and can vary from atom-pair distances to complex many-body (MB) descriptors. ${ }^{58}$ Independent of their dimensionality, however, descriptors are generally required to be translationally, rotationally, and permutationally invariant to guarantee a one to one mapping of energy to configuration. ${ }^{75}$

In this work, the PES of $\mathrm{hBN}$ is described by a sum of two-body (2B) terms and the high dimensional smooth overlap of atomic positions (SOAP $)^{76} \mathrm{MB}$ descriptor. Within SOAP, each atomic environment is represented by a local neighbour density generated by summing over Gaussians placed on all atoms within a certain cut-off. This density of the $i$ th atom, $\rho_{i}(\mathbf{r})$, is expanded in a basis set of radial functions $g_{n}(r)$ and spherical harmonics $Y_{l m}(\mathbf{r})$ as

$$
\rho_{i}(\mathbf{r})=\sum_{\substack{n<n_{\max } \\ l<l_{\max } \\|m| \leq l}} c_{n l m}^{i} g_{n}(r) Y_{l m}(\mathbf{r})
$$

wherein the related coefficients $c_{n l m}^{i}$ form the so-called SOAP vectors with $n, l$, and $m$ being the familiar integers. The product of two independent SOAP vectors represents the similarity between the related local environments, which can be easily made invariant to rotations. This so-called SOAP kernel is constructed for a certain set of training environments and the corresponding weight of their local contribution to the total energy of the global configurations is determined within the GKR fitting procedure. In addition to the energies, we also train on the atomic forces and virial stresses. A deeper insight and detailed derivation of the extension to use partial derivatives can be found elsewhere. ${ }^{69}$

Clearly, an accurate description of the PES is critically dependent on a comprehensive training set comprised of configurations of the desired phase space region. Here, this covers 
periodic and defect-free sheets and nanotubes of arbitrary chiralities. We generated a structural database containing more than 22,000 configurations. While most of them were extracted from AIMD trajectories, others originated from trajectories obtained with earlier GAP versions or were based on distinct displacements of atoms. In fact, including the latter configurations at $0 \mathrm{~K}$ is crucial to accurately predict the equilibrium configurations, lattice parameters or elastic constants. The number of atoms was limited to 200 and different thermodynamic conditions were sampled varying the temperature between 0 and $3000 \mathrm{~K}$ as well as applying uniaxial strain of up to $5 \%$ to layered hBN which should also enable the prediction of strained nanotubes. These conditions represent the region of phase space where the hBN-GAP can be safely applied and an accurate interpolation can be expected.

In practice, it is not desirable to use the entire structural database as training set due to the redundant information and the related increase of computational costs. Here a subset of $\approx 1600$ structures was used. These were selected through a combination of farthest point sampling ${ }^{59,75}$ and manual structure selection. While this has been shown to give an accurate representation of the original database, the training set could be further improved by using active learning which adds new configurations based on reliability of the model's prediction rather than the structural diversity in the existing training set ${ }^{77}$.

In order to obtain a qualitative overview of the complexity of problem we are attempting to fit, we performed a dimensionality reduction using sketch-map ${ }^{78,79}$ to visualise our structural database in two dimensional space. The resulting sketch-map, shown in figure 1 , clusters configurations based on their global similarity. Similar structures are located closely together while points with a high covariance are separated further apart. The clustering indicates the heterogeneity of the phase space region and provides useful information on the capabilities of the SOAP descriptor. For example, the sketch-map highlights the anticipated similarity between monolayers (Fig. 1D) and nanotubes with large diameter (Fig. 1C) while less stable thermally deformed nanotubes with high curvature are represented as an isolated island (Fig. 1B). Single layer and bulk hBN (Fig. 1G) are separated most far apart given the change of environment induced by the adjacent layers. This dissimilarity distance is bridged by bulk configurations with large interlayer spacing, strong thermal fluctuations (Fig. 1F) and bilayers (Fig. 1E). Interestingly, compressed bilayers (Fig. 1A) are singled out from the large clusters as well as sheared bulk configurations (Fig. 1H).

Having selected the training configurations, an appropriate choice of certain model parameters has to be made. Here, we will only discuss those parameters to which the model is most sensitive while a full overview is contained in table 1 and a more detailed explanation can be found elsewhere. ${ }^{69,73}$ Intuitively, cut-off and basis-set expansion of the SOAP vector have a high impact on the performance of the potential. We chose to truncate the spherical harmonic expansion after the eighth order, i.e. $l=8$ and $n=8$, and set the SOAP cut-off to $4.5 \AA$ which has been proven to be sufficient to reproduce the binding energy curve of graphite. ${ }^{72}$ However, this cut-off for the SOAP descriptor is too short to reproduce the interlayer interaction curve between $\mathrm{hBN}$ layers which does not tail off to zero until roughly $10 \AA$. Thus, we followed the procedure recently introduced $^{72}$ to fit a $2 \mathrm{~B}$-based model to the interaction curve using a cut-off of $10 \AA$. The predicted energy and forces were then subtracted during the actual fit where we used a shorter $2 \mathrm{~B}$ descriptor with the same cut-off as SOAP.

In practice, rather than using all local environments of the training set, the covariance matrix is constructed based on $M$ uncorrelated sparse points. ${ }^{73}$ Here, we chose 50 points for the 2B descriptor and 5000 for SOAP. The number of sparse points needed depends on the complexity of the target phase space region the 


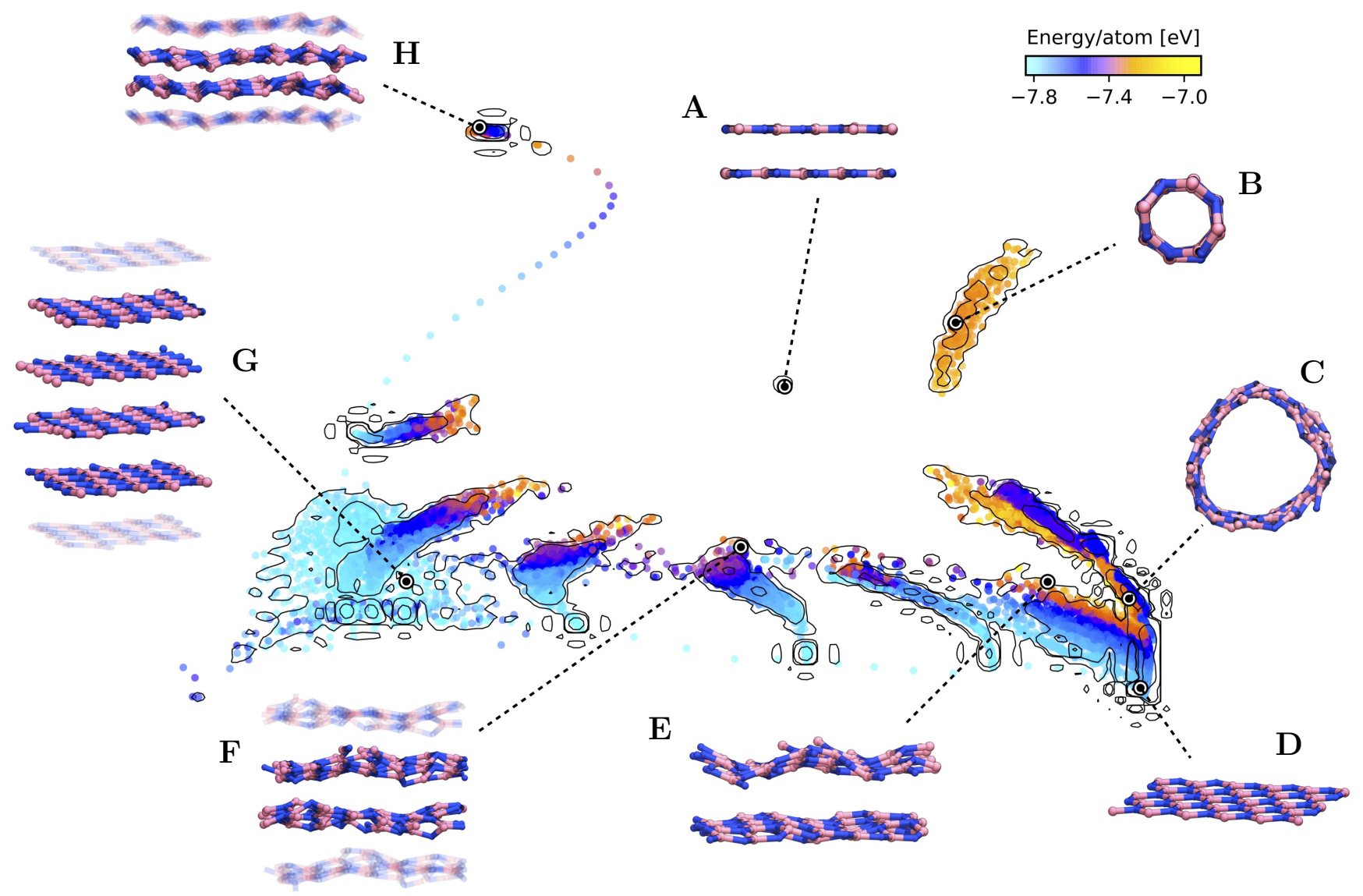

Figure 1: Sketch-map representation of the structural database generated for this work. Each point corresponds to one atomic configuration for which the local SOAP vectors are averaged and the SOAP kernel measures the global similarity between the structures. Short distances between points represent higher similarity while points far apart from each other express a strong structural deviation. The points are coloured according to their respective energy per atom calculated with DFT. Periodic images in z-direction are blurred to visualise bulk structures. (A) Compressed bilayer at $0 \mathrm{~K}$ (interlayer spacing $\approx 2.5 \AA) ;(\mathbf{B})$ Nanotube $(4,0)$ at $1000 \mathrm{~K} ;(\mathbf{C})$ Nanotube $(10,0)$ at $300 \mathrm{~K} ;(\mathbf{D})$ Monolayer at $30 \mathrm{~K} ;(\mathbf{E})$ Bilayer at $3000 \mathrm{~K} ;(\mathbf{F})$ Bulk at $3000 \mathrm{~K} ;(\mathbf{G})$ Bulk at $300 \mathrm{~K}$; (H) Compressed bulk at $700 \mathrm{~K}$.

model is fitted to. Given the focus on the defect-free hexagonal phase of BN, the selected number of sparse points for SOAP is in line with recent work. ${ }^{72}$ Besides these descriptor related parameters, the desirable target deviation, $\sigma$, of the fit to the target properties is a critical ingredient for an accurate model. While values which are too large result in poor agreement with the DFT reference per se, too small a chosen value can lead to overfitting depriving the GKR of its interpolation capability. Here, we used configuration-specific tolerances in order to distinguish between competing phases. For example, the PES of bulk hBN is very shallow close to the equilibrium resulting in energy differences of distorted cells of less than $1 \mathrm{meV}$. Thus, the tolerance was adapted for this kind of system while for high energy configurations larger values were set.

\section{Electronic structure calculations}

For each configuration, we conducted tightly converged DFT calculations to obtain the total energy, atomic forces, and virial stresses on which the GAP model was then trained. For all configurations we used the VASP plane-wave DFT code, ${ }^{80-83}$ using the Perdew-Burke-Ernz- 
Table 1: Model parameters for the hBNGAP. Target deviations $(\sigma)$ correspond to the range, with values depending on the specific configuration.

\begin{tabular}{|c|c|}
\hline 2b Descriptor & \\
\hline Cut-off $(\AA)$ & 4.5 \\
\hline Cut-off width $(\AA)$ & 0.5 \\
\hline$\delta$ & 0.5 \\
\hline Sparse method & Uniform \\
\hline Sparse points & 50 \\
\hline \multicolumn{2}{|l|}{ SOAP Descriptor } \\
\hline Cut-off $(\AA)$ & 4.5 \\
\hline Cut-off width $(\AA)$ & 0.5 \\
\hline$\delta$ & 0.1 \\
\hline Sparse method & CUR \\
\hline Sparse points & 5000 \\
\hline$l_{\max }$ & 8 \\
\hline$n_{\max }$ & 8 \\
\hline$\zeta$ & 2 \\
\hline \multicolumn{2}{|l|}{ Target Deviations } \\
\hline$\sigma_{\text {energy }}$ & $0.0001-0.0015$ \\
\hline$\sigma_{\text {force }}$ & $0.001-0.015$ \\
\hline$\sigma_{\text {virial }}$ & $0.005-0.05$ \\
\hline
\end{tabular}

erhof (PBE) functional ${ }^{84}$ with the DFT-D3 dispersion correction method ${ }^{85}$ employing the Becke-Johnson damping ${ }^{86}$, an energy cut-off of $1100 \mathrm{eV}$, a Gaussian smearing of $0.05 \mathrm{eV}$, and projector augmented wave pseudopotentials ${ }^{87,88}$. We chose PBE+D3 as it accurately predicts the out-of-plane lattice parameter of bulk hBN with respect to experiments. A comparison to other functionals is included in the supplementary information. The seemingly high plane-wave cut-off is based on convergence tests for the elastic properties of bulk hBN. The reciprocal lattice was sampled in periodic directions with a maximum distance between the $k$-points of $0.02 \AA^{-1}$ whereby the grid was either centered at the zone centre in case of hexagonal cells or Monkhorst-Pack based for orthorombic cells. For ionic and cell relaxations, cell shape and atomic positions were optimised independently until all forces were below $10^{-3} \mathrm{eV} \AA^{-1}$ and the total energy difference was converged to less than $10^{-6} \mathrm{eV}$. These settings were consistently used in this work if not stated otherwise.

\section{Molecular Dynamics Simulations}

The simulations of rippled graphene and hBN were performed in LAMMPS ${ }^{89}$ using a hexagonal simulation box comprising 7200 atoms applying a timestep of $1 \mathrm{fs}$. The unstrained sheets span a size of $15 \times 15 \mathrm{~nm}$ varying slightly according to the respective bond length. While this scale is beyond the usually accessible system size for DFT-based MD, GAP models can easily cope with these dimensions. First, we sampled the equilibrium lattice parameter of both materials at $300 \mathrm{~K}$ and zero external pressure in the isothermal-isobaric ensemble. A Nosé-Hoover chain thermostat and barostat was applied to ensure the target temperature and pressure. Then, we adjusted the systems' dimensions to sample the configurational space of the unstrained sheets in the canonical ensemble. In an analogous manner, we adapted the box dimensions to achieve relative strains between $-2.0 \%$ and $+2.0 \%$ to study the shape of mechanically induced ripples. The sampling time for all considered strains was roughly 0.5 ns.

\section{Results and Discussion}

\section{Evaluation of the hBN-GAP}

We validate the hBN-GAP and benchmark its predictive capabilities against its DFT reference, comparing it to other frequently used force fields, specifically the Tersoff potential ${ }^{31,32}$ parametrised by Sevik et al., ${ }^{39}$ the extended Tersoff potential (ExTeP) employing a modified bond-order parameter, ${ }^{41}$ and the ReaxFF ${ }^{42,43}$ parametrised by Weismiller et al. ${ }^{44}$ In addition to the standard versions, we also evaluated the predictions made by both Tersoff and ExTeP when combined with the recently developed interlayer potential (ILP). ${ }^{90-92}$ This coupling enables these models to account for the intermolecular interactions between hBN sheets. 
These are typically neglected by the independent bond-order potentials due to a cut-off of $2.0 \AA$ which is below the equilibrium separation between layers in bulk hBN of about 3.3 $\AA$. The list of force field models tested is by no means exhaustive and is merely meant to serve as baseline for our newly developed model in the context of previous work. This is also the case for the physical properties we evaluate the performance of the developed model on. We also note that all DFT comparisons employ the very same functional, PBE+D3, and electronic structure setup as applied in generation of training data. While this is essential to appropriately evaluate the quality of the constructed hBN-GAP, it might distort the benchmarking by implying that established force fields are in error when deviating from DFT results based on our chosen functional. To go some way to address this bias, we compared the performance of different functionals - local density approximation (LDA) and other generalised gradient approximations (GGA) for basic crystalline properties of hBN (see supplementary information for details).

The most intuitive measure to evaluate the performance of a ML potential is through force and energy errors on a basis of a validation set of configurations. This analysis has been carried out for most existing GAP models describing a great variety of materials. ${ }^{65,70,71}$ Force correlation plots as well as a comparison of computational costs for DFT, GAP, and the established models can be found in the supplementary information. Here, we will only report the root mean square errors (RMSE) with respect to DFT for the different models. For a validation set of 1450 randomly picked structures, which were not included in the training, the hBN-GAP achieves a RMSE of $0.09 \mathrm{eV} / \AA$. The established potentials are one order of magnitude less accurate with Tersoff, ExTeP, and ReaxFF yielding RMSEs of 1.09, 1.19 , and $6.17 \mathrm{eV} / \AA$, respectively. The ILP extended bond-order potentials perform surprisingly slightly worse with 1.11 and $1.22 \mathrm{eV} / \AA$ for Tersoff+ILP and ExTeP+ILP, respectively. While a correct reproduction of the atomic forces is a crucial requirement for any accurate interatomic potential, it is, however, not a sufficient criterion to secure an accurate description of the system of interest. In the course of this work, we observed that previously developed versions of our potential showed similar force errors but diverged significantly when comparing material properties. Moreover, high force errors do not lead to a bad description of macroscopic properties per se. Therefore, the evaluation presented will be focused on how the GAP model performs for energetic, geometrical, mechanical, and vibrational properties of different hBN phases.

The reproduction of lattice parameters for different phases is an essential requirement an accurate potential must fulfil. A comprehensive comparison of lattice parameters predicted by the hBN-GAP and other force fields as well as the relative deviation from DFT can be found in table 2. The DFT results agree well with experimental results ${ }^{93}$ for the monolayer and bulk phase. Overall, our model performs very well with respect to its DFT reference for all lattice lengths and structures with an average error of $0.10 \%$.

The established force fields considered also achieve a very good performance for the geometric properties albeit being slightly less accurate than our hBN-GAP. Tersoff and ExTeP yield average errors of $0.42 \%$ and $0.34 \%$, respectively, although their actual score is somewhat biased as no out-of-plane lengths were considered. Coupling both potentials tuned with ILP, however, allows for the description of multilayered $\mathrm{hBN}$ and they both yield an average error of $1.57 \%$ and $1.59 \%$, respectively. ReaxFF achieves a sufficient accuracy for the in-plane and nanotubes lattice parameters but it fails dramatically to capture the out-of-plane lengths resulting in an overall error of $4.06 \%$.

In addition to the structural characteristics, we now consider the energetics of the relevant phases as shown in figure 2. To this end, the computed formation energies of the optimised structures are given per atom and relative to 
Table 2: Comparison of lattice parameters and interlayer distances for the different hBN phases between DFT (PBE+D3), the hBN-GAP, and other force fields. The lattice parameters are given in absolute values while the relative error with respect to DFT is given in percentage in brackets. For bulk hBN the out-of-plane lattice parameter c corresponds to the length of the respective lattice vector while for an isolated bilayer $\tilde{\mathbf{c}}$ is defined as the interlayer distance. Due to the short cut-off of Tersoff and ExTeP no values (-) are given for the out-of-plane lengths of bilayer and bulk hBN.

\begin{tabular}{|c|c|c|c|c|c|c|c|}
\hline & \multicolumn{7}{|c|}{ Lattice Parameter $[\AA]$ (\% Error) } \\
\hline & DFT & GAP & Tersoff & Tersoff + ILP & ExTeP & ExTeP + ILP & ReaxFF \\
\hline Bulk hBN (a) & 2.51 & $2.51(0.0)$ & $2.50(0.4)$ & $2.50(0.4)$ & $2.50(0.4)$ & $2.50(0.4)$ & $2.55(1.6)$ \\
\hline Bulk hBN (c) & 6.61 & $6.60(0.2)$ & - & $6.46(2.3)$ & - & $6.45(2.4)$ & $5.88(11.0)$ \\
\hline Bilayer (a) & 2.51 & $2.51(0.0)$ & $2.50(0.4)$ & $2.50(0.4)$ & $2.50(0.4)$ & $2.50(0.4)$ & $2.55(1.6)$ \\
\hline Bilayer $(\tilde{\mathrm{c}})$ & 3.36 & $3.36(0.0)$ & - & $3.26(3.0)$ & - & $3.26(3.0)$ & $2.99(11.0)$ \\
\hline Monolayer (a) & 2.51 & $2.51(0.0)$ & $2.50(0.4)$ & $2.50(0.4)$ & $2.50(0.4)$ & $2.50(0.4)$ & $2.55(1.6)$ \\
\hline Nanotube $(10,0)$ & 4.34 & $4.32(0.5)$ & $4.32(0.5)$ & $4.32(0.5)$ & $4.32(0.5)$ & $4.32(0.5)$ & $4.34(0.0)$ \\
\hline Nanotube $(10,10)$ & 2.51 & $2.51(0.0)$ & $2.50(0.4)$ & $2.50(0.4)$ & $2.51(0.0)$ & $2.50(0.4)$ & $2.55(1.6)$ \\
\hline
\end{tabular}

bulk hBN which is the thermodynamically most stable phase. In the case of layered $\mathrm{hBN}$, the observations from the lattice parameters for Tersoff and ExTEP are confirmed. Due to the lack of non-bonded contributions to the energy these potentials cannot distinguish between different numbers of sheets yielding an RMSE of more than 54 and $67 \mathrm{meV} /$ atom, respectively. Compensating for this with the ILP, the tuned Tersoff and ExTeP potentials achieve very good agreement with DFT with an improved RMSE of 4 and $26 \mathrm{meV} /$ atom, respectively. While the performance of ReaxFF is mediocre with an error of $45 \mathrm{meV} /$ atom, our hBN-GAP achieves also very good agreement with DFT with a RMSE of $3 \mathrm{meV} /$ atom. This high accuracy is persistent throughout the tested configurations including the energetically less favourable nanotubes irrespective of diameter or chirality. To avoid confusion it is worth mentioning that the total energy of the nanotubes predicted by Tersoff and ExTeP is identical to those predicted by their versions enhanced by ILP. The energy shift in the plot is caused by the change of the energy for the reference bulk hBN.

Part of the attention garnered by $\mathrm{hBN}$ is due to its excellent mechanical properties. More- over, a system's response to mechanical compression and tension is an essential property for 2D materials as it affects the shape of ripples in atomically thin sheets. ${ }^{21,94}$ In order to ensure an accurate description of the straindeformation relationship we computed the relevant elastic constants of bulk hBN with DFT, GAP, and existing potentials. An overview of these results and a comparison to experimental measurements ${ }^{95}$ can be found in table 3. In order to determine the elastic constants, finite distortions were made to the optimised cell and the ions were allowed to relax. The respective constants were then calculated from the stress-strain relationship whereby different magnitudes of strain were applied to guarantee a linear behaviour. For ReaxFF this linear relationship could not be achieved. Instead physically unrealistic and negative elastic constants were obtained. This indicates that the parametrisation of the ReaxFF used ${ }^{44}$ does not yield a satisfactory description of the equilibrium shape of bulk $\mathrm{hBN}$, namely $\mathrm{AA}^{\prime}$ stacking in a hexagonal unit cell. This issue could not be solved by changing to a different parameter set $^{46}$ for ReaxFF. The results for ReaxFF are, thus, not included in table 3 . 
Table 3: Mechanical properties of bulk hBN calculated with DFT (PBE+D3), the hBN-GAP, and other force fields in comparison to experimental measurements. ${ }^{95}$ For the experiments the values in parenthesis correspond to the experimental uncertainty. Due to the short cut-off of Tersoff and ExTeP no values (-) are given for the out-ofplane elastic constants $\mathrm{C}_{13}, \mathrm{C}_{33}, \mathrm{C}_{44}$, and the bulk modulus.

\begin{tabular}{lccccccc}
\hline & \multicolumn{6}{c}{ Elastic constants [GPa] } \\
\cline { 2 - 8 } & DFT & GAP & Tersoff & Tersoff + ILP & ExTeP & ExTeP + ILP & Experiment $^{95}$ \\
\hline $\mathrm{C}_{11}$ & 881.8 & 853.0 & 969.9 & 858.0 & 849.1 & 861.9 & $811.0(12.0)$ \\
$\mathrm{C}_{12}$ & 195.6 & 216.8 & 303.0 & 269.8 & 152.4 & 156.3 & $169.0(24.0)$ \\
$\mathrm{C}_{13}$ & 0.0 & 12.9 & - & 2.8 & - & 2.8 & $0.0(3.0)$ \\
$\mathrm{C}_{33}$ & 28.9 & 36.1 & - & 37.9 & - & 37.7 & $27.0(5.0)$ \\
$\mathrm{C}_{44}$ & 3.3 & 0.3 & - & 6.5 & - & 6.5 & $7.7(5.0)$ \\
$\mathrm{B}$ (eq) & 27.4 & 35.4 & - & 35.9 & - & 35.4 & $25.6(8.0)$ \\
$\mathrm{B}$ (MEoS) & 31.2 & 34.7 & - & 39.8 & - & 39.7 & \\
\hline
\end{tabular}

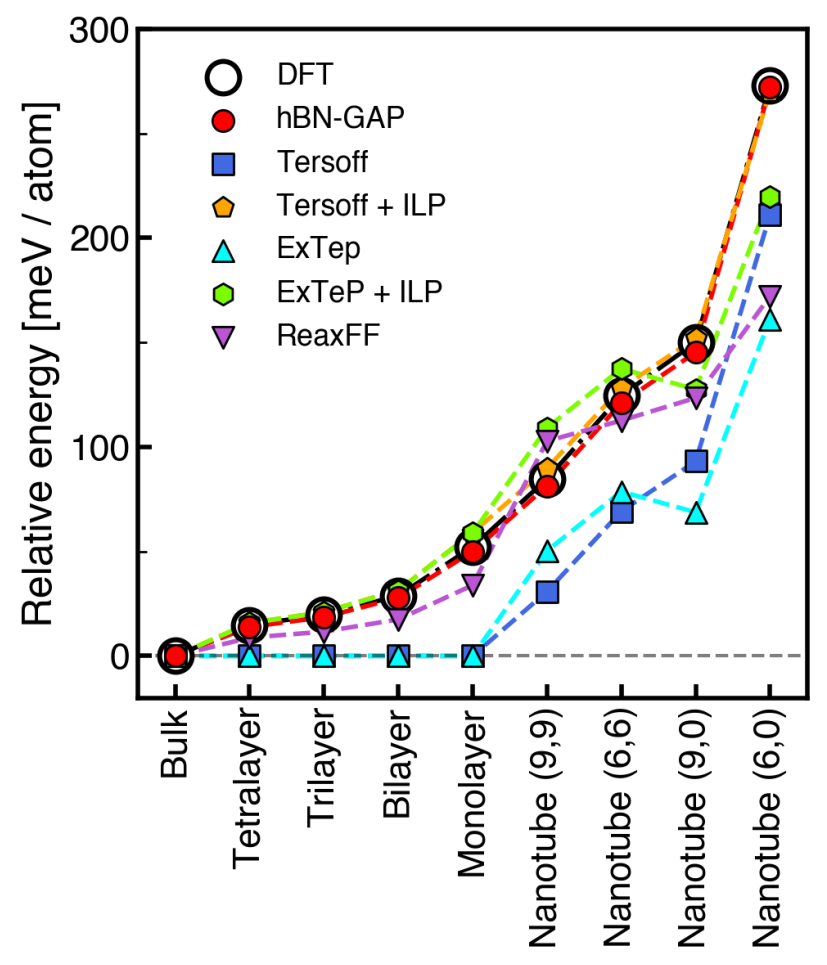

Figure 2: Formation energies of the hBN configurations computed with DFT (PBE+D3), the hBN-GAP, and other established force fields. Both bond-order potentials Tersoff and ExTeP are shown with and without the correction for multiple layers contributed by the ILP. All energies are plotted relative to bulk hBN. The lines are a guide to the eye.

Our DFT calculations match the measurements for most constants within the experi- mental uncertainty. Both approaches predict that there is no coupling between in-plane stress (strain) and out-of-plane strain (stress), i.e. $\mathrm{C}_{13}=0$. Our hBN-GAP achieves a very good agreement with its reference DFT, particularly for the in-plane constants $\mathrm{C}_{11}, \mathrm{C}_{12}$ and the out-of-plane coupling $\mathrm{C}_{33}$ which also corresponds to a high accuracy of the interlayer binding curve. ${ }^{96}$ The prediction for $\mathrm{C}_{13}$ and $\mathrm{C}_{44}$ is less accurate while the actual behaviour, i.e. a weak coupling between stress and strain in the respective directions, is still captured qualitatively. It is worth noting, that very tight target deviations for the virials $\sigma_{\text {virial }}$ are required to obtain very high accuracy for these quantities, however, using such target deviations can lead to overfitting. Beyond the individual couplings between different directions, a correct description of the overall compressibility of the material, i.e. the bulk modulus $\mathrm{B}$, is desirable. Here, we calculate $\mathrm{B}$ via a relationship ${ }^{97}$ dependent on the elastic constants,

$$
\mathrm{B}=\frac{\mathrm{C}_{33}\left(\mathrm{C}_{11}+\mathrm{C}_{12}\right)-2\left(\mathrm{C}_{13}\right)^{2}}{\mathrm{C}_{11}+\mathrm{C}_{12}+2 \mathrm{C}_{33}-4 \mathrm{C}_{12}},
$$

and by fitting the Murnaghan equation of state $(\mathrm{MEoS})^{98}$ to the energy-volume curve. For the latter approach, the anisotropic character of $\mathrm{hBN}$ requires a non-isotropic compression to avoid erroneous results. As shown in table 3 , both methods yield similar results and the 
hBN-GAP agrees well with DFT.

Evaluating the performance of established potentials, both Tersoff and ExTeP suffer from their unsatisfactorily short cut-off predicting infinite compressibility for any reasonable geometry. Therefore, no values are included for elastic constants dependent on out-of-plane strain. By applying the ILP correction both tuned potentials perform very well compared to DFT and experiments. While they are in average sligthly less accurate for the in-plane constants they show an even better agreement for $\mathrm{C}_{13}$ and $\mathrm{C}_{44}$ than the hBN-GAP. Despite this good performance, it should be noted that the high accuracy seems to be mainly based on the ILP correction. Therefore, doubts must be cast on the predictive capability of these potentials for isolated $\mathrm{hBN}$ sheets and nanotubes as the ILP has no contribution to the potential energy in these cases.

For low-dimensional materials, it has been shown that phonon modes affect adsorbate (notably water) diffusion through carbon nanotubes $^{27}$ and across graphene. ${ }^{99}$ Other relevant thermodynamic properties, such as heat capacity, thermal conductivity, and thermal expansion coefficients are also closely linked to phonons. Therefore, an accurate description of the dynamics of the lattice is an essential requirement for the $\mathrm{hBN}-\mathrm{GAP}$.

Before discussing the performance of the hBN-GAP, it is important to emphasise some essential aspects in the calculation of the phonon dispersion curves for hBN. Due to the material's polar nature, a dipole is induced by the longitudinal optical (LO) phonon modes which creates a macroscopic electric field affecting the dispersion behaviour of the optical phonons in the long wavelength limit, i.e. $q \rightarrow 0$. This complicates the determination of phonon dispersion curves within the so-called

\footnotetext{
${ }^{1}$ As the D3 dispersion correction is not implemented in the DFPT framework of the QE software package, the phonon calculations of the hBN monolayer and nanotube are done using the PBE functional without dispersion correction.
}

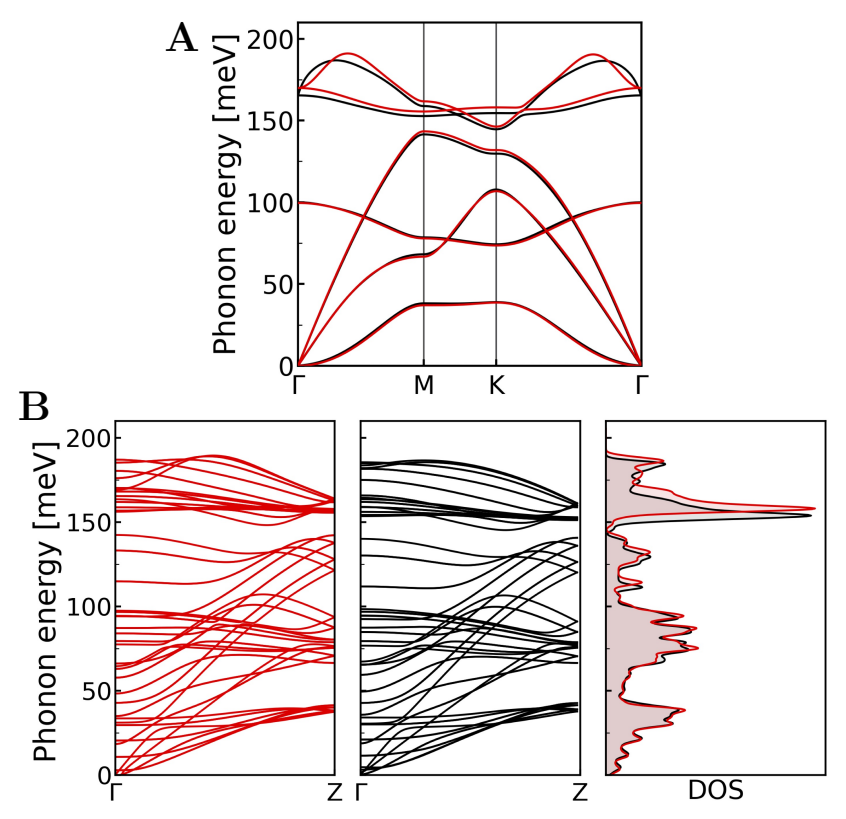

Figure 3: Phonon dispersion curves calculated for different phases of hBN. The black data corresponds to the DFT reference ${ }^{1}(\mathrm{PBE})$ while the red data represents our hBN-GAP. (A) Phonon dispersion for an isolated hBN sheet. (B) Phonon dispersion for a selected nanotube with chirality $(6,6)$ as well as the density of states (DOS) predicted with GAP and DFT. Due to the number of bands, the phonon dispersion for other force fields are included in the supplementary information.

finite displacement method requiring an infinitely large supercell ${ }^{100}$ or the addition of a non-analytical correction to the dynamic matrix based on the Born effective charges ${ }^{101}$ as shown by Gonze et al. 102,103. Alternatively, the analytical part of the dynamical matrix is also readily calculated by the linear response of the electron density providing the entire dispersion curve based on the unit cell only. ${ }^{104}$

Here, as first-principle reference we conduct density functional perturbation theory (DFPT) calculations because an appropriate treatment of the Coulombic interactions for 2D materials has recently been implemented ${ }^{105}$ in the DFPT framework in the QUANTUM ESPRESSO (QE) ${ }^{106,107}$ software package. Equivalent setups for the DFPT and DFT calculations conducted with QE and VASP, respectively, were 
used. We ensured a high agreement between both codes throughout different properties and a more detailed comparison can be found in the supplementary information. For GAP and other potentials the phonon dispersion curves are calculated using the finite displacement method provided by the Phonopy package. ${ }^{108}$ For potentials treating charges explicitly, contributions due to the non-analytical correction according to Gonze et al. ${ }^{102,103}$ can be added. This, however, does not apply for the bondorder potentials and the hBN-GAP.

In this work, we compare the phonon dispersion of a hBN single layer and a hBN nanotube with chirality $(6,6)$. Very good agreement was obtained between our hBN-GAP and inelastic X-ray scattering (IXS) measurements ${ }^{109-112}$ for bulk hBN which is reported in the supplementary information. The dispersion curves computed with DFPT and hBN-GAP are shown in figure 3 and the performance of other force fields can be found in the supplementary information. As shown in figure 3A the GAP achieves a very good agreement with its DFT reference, particularly for the acoustic modes. The predicted frequencies of the optical branches at the $\Gamma$-point are correct to $<5 \mathrm{meV}$. GAP is not able to reproduce the finite slope of the LO dispersion close to the zone center. The generated electric field causes a non-zero slope which can be captured by adding the non-analytical correction to the dynamic matrix as described above. ${ }^{105}$ As charges and related electrostatics are treated implicitly, the current version of the hBN-GAP cannot reproduce this characteristic behaviour. It is worth noting, however, that explicit charges can be incorporated in the GAP framework which will be part of future extensions of the hBN-GAP.

The good performance of the hBN-GAP for the vibrations in monolayer $\mathrm{hBN}$ becomes even more apparent when comparing to existing force fields shown in the supplementary information. Both the Tersoff and ExTeP potentials provide a good though less accurate description of the acoustic modes yielding deviations of more than $20 \mathrm{meV}$. Further, the phonon energy of the optical modes, particularly LO and $\mathrm{TO}$, is overestimated by up to $40 \mathrm{meV}$ for both potentials.

To understand the performance of the hBNGAP for a $(6,6)$ nanotube, we focus on the overall phonon density of states (DOS) rather than individual bands due to the large number of atoms in the unit cell. As depicted in figure $3 \mathrm{~B}$, the hBN-GAP reproduces the DFT results very well showing only small deviations of typically $<5 \mathrm{meV}$. The DOS predicted by the hBN-GAP is almost congruent with the one obtained from DFT calculations except for a small offset for high energy modes. However, these deviations are in the same order of magnitude as the difference between predictions made by different DFT functionals ${ }^{113}$. For the established force fields we observe a similar trend to layered $\mathrm{hBN}$. While the low energy modes are described sufficiently accurately yielding deviations of about $7 \mathrm{meV}$, the high energy modes shifted by more than $30 \mathrm{meV}$ for both Tersoff and ExTeP potential (see supplementary information).

\section{Application to rippling in $\mathrm{hBN}$ and graphene}

After the careful validation in the previous section, we focus on the application of the $\mathrm{hBN}-\mathrm{GAP}$ to investigate rippling in single layer hBN in comparison to graphene. Although their name may suggest otherwise, these $2 \mathrm{D}$ materials are not perfectly flat at finite temperature but exhibit local height fluctuations. ${ }^{21,26,56,114-117}$ These thermally activated ripples are an intrinsic feature of $2 \mathrm{D}$ crystals which are stabilised by an anharmonic coupling between bending and stretching modes. ${ }^{20,94,118-120}$ Moreover, the corrugation of the surface can strongly affect the material's properties ${ }^{22-24}$ indicating a close link between atomic and electronic structure. The behaviour of the $2 \mathrm{D}$ crystal is, thus, directly affected by the average amplitude of the height fluctuations, $\langle h\rangle$, which, according to the theory of flexible membranes, ${ }^{118-120}$ scales with the sys- 
tem size, $L$, as $\langle h\rangle \propto L^{1-\eta / 2}$, with $\eta$ being the anomalous rigidity exponent. While the results of atomistic simulations on graphene ${ }^{56}$ and a general nonperturbative renormalisationgroup approach ${ }^{121}$ suggest the transferability of $\eta=0.85$, yet, a smaller value of $\eta \approx 0.66$ was obtained for hBN ${ }^{41}$ based on molecular dynamics simulations using the ExTeP model. This deviation, however, may stem from simulation settings, particularly, too small length and time scales. While system sizes required to confirm this assumption exceed 50000 atoms and are, thus, beyond the scope of this work, valuable insight on the predictive capability of the hBNGAP can be gained based on smaller systems comprising 7200 atoms.

Here, we first utilise the hBN-GAP to determine $\eta$ based on MD simulations following the procedure described by Los et al. ${ }^{56}$ For the sake of comparability, we also compute $\eta$ for graphene using the same methodology whereby the interatomic interactions are described by the graphene-GAP potential. ${ }^{70}$ However, as the actual shape and amplitude of the ripples can be strongly altered by externally introduced strain, ${ }^{26,99,117}$ we subsequently analyse the impact of tension and compression on the mean amplitude and phase behaviour of both materials.

The scaling exponent $\eta$ is computed based on the comparison between the results of our atomistic simulations and the prediction of the normal-normal correlation $G(q)$ function provided by the theory of flexible membranes. ${ }^{118-120}$ A general expression for $G(q)$ is given by the Dyson equation

$$
G^{-1}(q)=G_{0}^{-1}(q)+\Sigma(q),
$$

where $G_{0}(q)$ represents the harmonic approximation,

$$
G_{0}(q)=\frac{T N}{\kappa S_{0} q^{2}},
$$

and $\Sigma(q)$ is the self energy accounting for the anharmonic coupling at small wavevectors,

$$
\Sigma(q)=\frac{A S_{0}}{N} q^{2}\left(\frac{q_{0}}{q}\right)^{\eta},
$$

with $q$ as length of the $2 \mathrm{D}$ wavevector $\mathbf{q}, T$ as temperature in energy units, $N$ as number of atoms, $\kappa$ as bending rigidity, $S_{0}$ as area per atom, A as an unknown prefactor, $q_{0}=$ $2 \pi \sqrt{B / \kappa}$, B as 2D bulk modulus, and $\eta$ as the desired scaling exponent. In the limit of slowly varying height fluctuations $\left(|\nabla h|^{2} \ll 1\right)$, $G(q)$ as defined in equation 3 is identical to $q^{2}\left\langle|h(q)|^{2}\right\rangle$, where $h(q)$ represents the Fourier transform of the atomic out-of-plane displacement, $h(\mathbf{x})$, which is directly obtained from a MD trajectory. ${ }^{21,56,114}$ In practice, however, $h(\mathbf{x})$ is smoothed by averaging over the nearest neighbours before numerically calculating the Fourier components. ${ }^{114}$ Further, the wavevectors are restricted by the system size to a minimum length of $2 \pi / L$ corresponding to $\approx 0.048$ $\AA^{-1}$ for our system, which has been shown to be sufficiently small to access the anharmonic region. ${ }^{56}$

Figure 4 depicts $G(q) / N$ calculated from the MD trajectories for unstrained $\mathrm{hBN}$ and graphene. While for large wavevectors the continuum theory breaks down, $G(q)$ is accurately reproduced by the harmonic approximation $G_{0}(q)$ for wavevectors in range $q^{*} \lesssim q \lesssim$ $1 \AA^{-1}$ where $q^{*}$ is given by the Ginzburg criterion $^{120}$

$$
q^{*}=\sqrt{\frac{3 T B}{8 \pi \kappa^{2}}},
$$

quantifying the long-wavelength limit where anharmonic contributions become dominant. We extracted the bending rigidity $\kappa$ at $300 \mathrm{~K}$ for both materials by comparing equation 4 to the simulation results for wavevectors $0.3<q<1 \AA^{-1}$ yielding $\kappa=1.09 \mathrm{eV}$ and $\kappa=1.65 \mathrm{eV}$ for $\mathrm{hBN}$ and graphene, respectively. As these values seem very high in comparison to previous work using classical potentials, ${ }^{21,56,115}$ we computed $\kappa$ at $0 \mathrm{~K}$ based on the out-of-plane acoustic phonon branch $(\mathrm{ZA})^{122}$ for both GAP models. The results of $\kappa=0.90 \mathrm{eV}$ and $\kappa=1.58 \mathrm{eV}$ for $\mathrm{hBN}$ and 


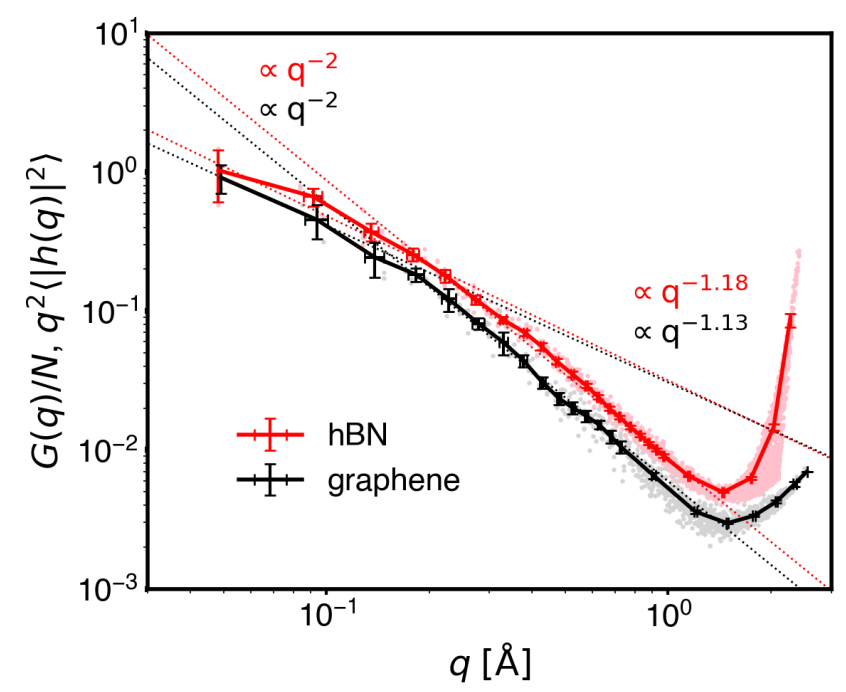

Figure 4: Normal-normal correlation function $G(q) / N$ calculated for graphene and $\mathrm{hBN}$ based on MD simulations using the hBN-GAP and the graphene-GAP, respectively. The pale markers correspond to the numerical results directly obtained from our simulations. This data was then smoothed and averaged within small intervals of wavevectors which is represented by the continuous line including error bars. The dotted lines imply the asymptotic behaviour with power-law $q^{-2}$ for the harmonic approximation for large $q$ and $q^{-(2-\eta)}$ for long wavelengths where the anharmonic contributions dominate. For graphene and $h B N$ values of $\eta=0.87$ and $\eta=0.82$, respectively, are obtained

graphene, respectively, agree very well with previous DFT calculations. ${ }^{123,124}$

We now focus on the long-wavelength simulation data in the range $q \leq q^{*}$ which we compare to the anharmonic self energy $\Sigma(q)$ given by equation 5 . Due to the rather weak temperature dependence of the $2 \mathrm{D}$ bulk modulus $B,{ }^{125}$ we used the predicted values of the GAP models for $0 \mathrm{~K}$ which are $B=11.38 \mathrm{eV} / \AA^{2}$ and $B=13.59 \mathrm{eV} / \AA^{2}$ for $\mathrm{hBN}$ and graphene, respectively. Further, to be comparable with previous work ${ }^{21,56}$ we fixed the prefactor $A=1$. This way, a best fit to the simulation data yields scaling exponents of $\eta=0.82$ for $\mathrm{hBN}$ and $\eta=0.87$ for graphene which agree very well with established findings. ${ }^{56,121}$
hlAs pointed out above, the small deviations between the materials might be caused by the limited availability of fitting data in the longwavelength range requiring significantly larger system sizes. Our results, therefore, confirm the observation made by Los et al. ${ }^{56}$ that the scaling exponent is independent of the bending rigidity $\kappa$. Conversely, the distinct materials' properties strongly affect the actual height of the ripples where small values of $\kappa$ result in larger height fluctuations as shown by the shift of hBN in figure 4. Also, according to equation 6 the crossover between the harmonic and anharmonic regime is switched to lower $q$ for lower values of $B / \kappa^{2}$ which is also confirmed by our results.

So far, we have shown that the scaling behaviour of ripples is predicted to be almost identical in hBN and graphene while the amplitude of the out-of-plane deviations for a given system size will be slightly larger in $\mathrm{hBN}$ than in graphene. However, these findings apply in the limit of no external strain. To go beyond this, we analyse the average height of these mechanically induced ripples based on MD trajectories of hBN and graphene exposed to different intensities of strain. To this end, we determined the atom which shows the highest deviation from the centre mass perpendicular to the flat sheet. This measure is then averaged over the entire trajectory and the respective error based on block averages was computed to quantify the uncertainty. We also evaluated the influence of the box geometry on the shape and amplitude of the ripples in compressed systems. While the shape changes significantly from a hexagonal to an orthorombic simulation box the magnitude of the height fluctuations is almost unaffected (see supplementary material).

In analogy to our findings for the unstrained systems, ripples in hBN and graphene show a very similar reaction to strain as shown in figure 5A. The measured amplitudes agree with previous simulation work based on established force fields for both graphene ${ }^{99}$ and hBN. ${ }^{126}$ Throughout the entire strain range, hBN shows a larger average rippling height than graphene 


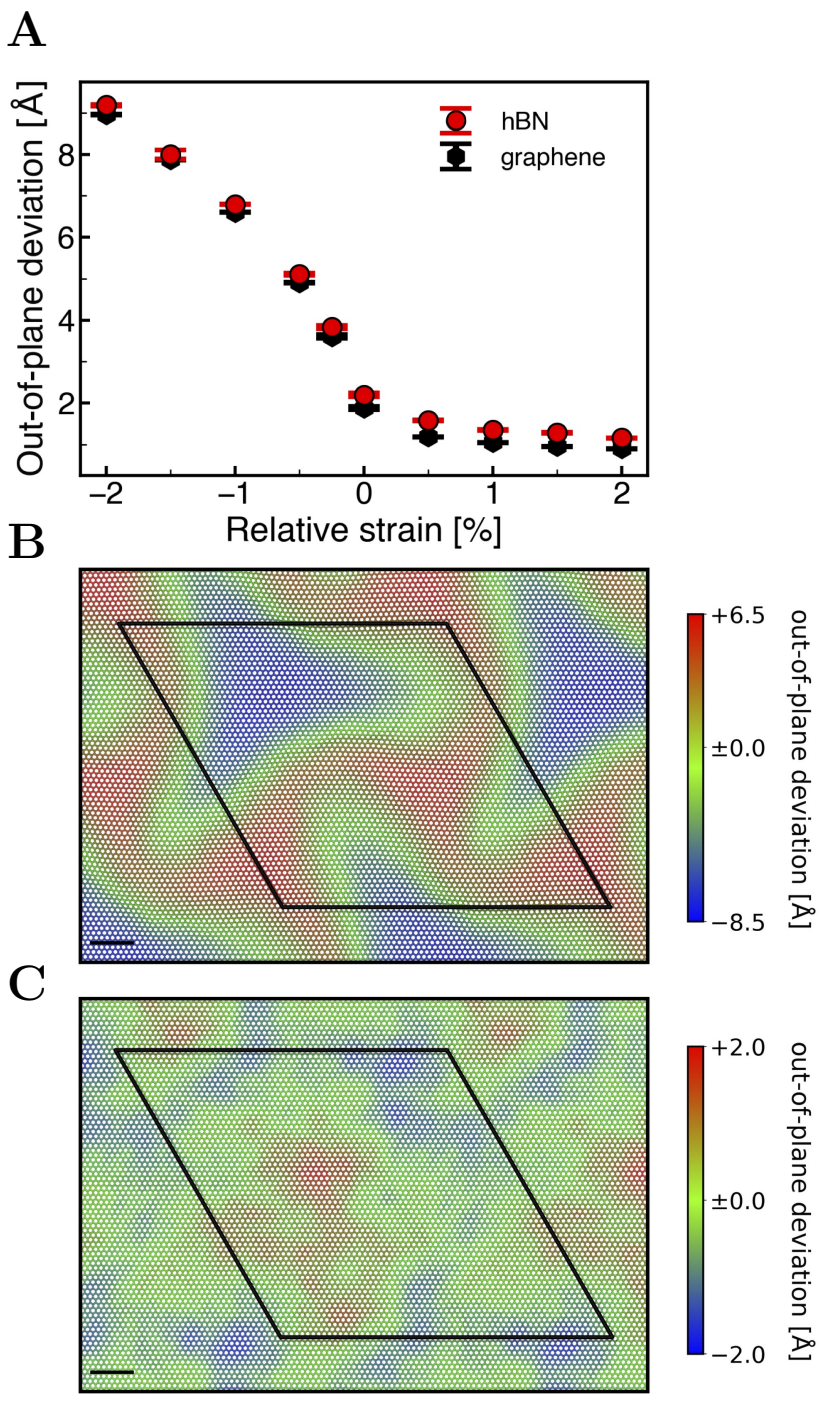

Figure 5: (A) Averaged out-of-plane deviation as function of applied strain for hBN (red) and graphene (black) predicted by the hBNGAP and graphene GAP, respectively.(B-C) Snapshot of the MD simulations for an unstrained and $\mathrm{hBN}$ monolayer and compression of $-2.0 \%$. The atoms are coloured according to their displacement from the center of mass in z-direction. The simulation box is indicated in black and the scale bar in the bottom left represents $2 \mathrm{~nm}$.

due to its lower bending rigidity $\kappa$. Large negative strains lead to a compression of the $2 \mathrm{D}$ sheets as the system stabilises by deforming in the third dimension while uniaxial tension causes the amplitude of the out-of-plane deformations to shrink. We observed a phase transition from short-lived fluctuations (figure
5B) to spatially coherent soliton-like ripples (figure 5C) which takes place between a strain of $-0.25 \%$ and $-0.5 \%$ for both materials. This is in line with simulations performed on isolated graphene sheets. ${ }^{99}$ Interestingly, for multilayered graphene previous simulations ${ }^{127}$ have led to the suggestion that a much higher strain of $-2.8 \%$ is required to induce spatially coherent ripples. Exploring this issue for $\mathrm{hBN}$ and the impact of vdW forces between multiple layers on the phase transition of ripples is a fascinating field and will be subject of future work.

Finally, it is worth mentioning that these studies on ripples show that the hBN-GAP proves to be transferable to system sizes outside both the training set and the accessible scale of DFT. Indeed, the predicted phase transition from figure $5 \mathrm{~B}$ to a rippling pattern as in figure $5 \mathrm{C}$ is exclusively based on an interpolation between the local environments in the training set.

\section{Conclusion}

In this work, we introduced a machine learningbased atomistic model to treat different phases of $\mathrm{hBN}$ ranging from isolated sheets to the bulk phase and nanotubes of any chirality. Our model was constructed using the GAP methodology and was trained on energies, forces, and virial stresses obtained from tightly converged vdW-inclusive DFT calculations. We benchmarked the hBN-GAP against its DFT reference and other established potentials and demonstrated its capabilities through a variety of tests including the elastic constants and the phonon dispersion spectra for nanotubes and layered hBN. Based on this evaluation we believe to have developed an accurate model, however, we do not claim perfect accuracy for each property. Rather, we acknowledge that the prediction for individual properties may be compromised due to the variety of configurations and thermodynamic conditions considered.

We applied our model as part of a compar- 
ative analysis of thermally and mechanically induced rippling in graphene and $\mathrm{hBN}$. In the course of this study, we found that the bending rigidities predicted by both GAP models are significantly larger than reported by previous work based on established force fields. Both models predict an almost identical scaling behaviour of the rippling height with $\eta \approx 0.85$ for $\mathrm{hBN}$ and graphene being in accordance with the theory of flexible membranes. In contrast to the scaling behaviour, however, the actual height of the out-of-plane deviations for a given system size is directly affected by the material's resistance to bending showing larger values for hBN. This observation also holds for mechanically deformed ripples irrespective of whether the system was exposed to compression or tension. Eventually, the phase transition from randomly fluctuating to soliton-like ripples occurs at roughly the same strain rate for both materials.

It is important, however, to point out some of the limitations and shortcomings of our potential. Despite being computationally several orders of magnitude more efficient than DFT, the evaluation of the SOAP descriptor is demanding and the hBN-GAP is roughly three orders of magnitude more expensive than the established hBN force fields. Although we extended the range of the $2 \mathrm{~B}$ descriptor to $10 \AA$, our model is constrained by its finite cut-off and is, thus, not able to appropriately treat long-range interactions due to electrostatics or dispersion. Further, treating charges only implicitly prevents a correct description of the phonon dispersion curves at small wavevectors. Additionally, in reality hBN is inevitably found with defects and dopants are incorporated in the crystal's lattice. ${ }^{128}$ While we did not consider such systems in this study, they will be part of future extensions of our training set and GAP model.

Looking forward, future applications of the hBN-GAP could involve the study of how rippling is affected by dispersion interactions in bilayer and multilayer hBN. Another application may include treating our model as struc- ture generator for rippled sheets to investigate the impact of the corrugation on proton transfer ${ }^{52,129}$ and the adsorption and, potentially, permeation of small atoms and molecules with DFT. ${ }^{130,131}$ Moreover, the hBN-GAP can be readily combined with any other force field and, thus, could be used to study the interface with fluids such as liquid water. In this context, a high resolution of the phonon modes, as achieved with our model, is essential as adsorbate motion on layered materials, and through nanotubes couples with phonon modes of the solid. ${ }^{27-30}$

We have made our potential as well as training and evaluation sets freely available at [http: //www.libatoms.com]. The hBN-GAP can be readily applied within the QUIP software package and used to run MD simulations in LAMMPS. ${ }^{89}$

\section{Supporting Information Avail- able}

The Supporting Information is available free of charge on the ACS Publications website at DOI:

Comparison of DFT functionals.

Benchmarking of the hBN GAP including formation energies of nanotubes, the binding curve in bulk hBN, as well as the computational efficiency.

Phonon dispersion curves predicted by the various force fields.

Analysis of the impact of box shape and box size on the predicted rippling amplitudes.

Acknowledgement We thank Gábor Csányi for fruitful discussions and valuable advice during the fitting process of the GAP model. We also thank Piero Gasparotto for his guidance and support in creating the sketch-map. The authors are grateful to the UK Materials and Molecular Modelling Hub for computational resources, which is partially funded 
by EPSRC (EP/P020194/1). Via our membership of the UK's HEC Materials Chemistry Consortium, which is funded by EPSRC (EP/L000202, EP/R029431), this work used the ARCHER UK National Supercomputing Service (http://www.archer.ac.uk). Additionally, we are also grateful for the use of the UCL Grace High Performance Computing Facility (Grace@UCL), and associated support services, in the completion of this work. Similarly, we are also grateful for the use of the Imperial College Research Computing Service, DOI: $10.14469 / \mathrm{hpc} / 2232$.

\section{References}

(1) Watanabe, K.; Taniguchi, T.; Kanda, H. Direct-bandgap properties and evidence for ultraviolet lasing of hexagonal boron nitride single crystal. Nature Materials 2004, 3, 404-409.

(2) Chen, Y.; Zou, J.; Campbell, S. J.; Caer, G. L. Boron nitride nanotubes: Pronounced resistance to oxidation. $A p$ plied Physics Letters 2004, 84, 24302432 .

(3) Pakdel, A.; Bando, Y.; Golberg, D. Nano boron nitride flatland. Chemical Society Reviews 2014, 43, 934-959.

(4) Geim, A. K.; Grigorieva, I. V. Van der Waals heterostructures. Nature 2013, 499, 419-425.

(5) Britnell, L.; Gorbachev, R. V.; Jalil, R.; Belle, B. D.; Schedin, F.; Mishchenko, A.; Georgiou, T.; Katsnelson, M. I.; Eaves, L.; Morozov, S. V. et al. FieldEffect Tunneling Transistor Based on Vertical Graphene Heterostructures. Science 2012, 335, 947-951.

(6) Xia, F.; Wang, H.; Xiao, D.; Dubey, M.; Ramasubramaniam, A. Two-dimensional material nanophotonics. Nature Photonics 2014, 8, 899-907.

(7) Lei, W.; Portehault, D.; Liu, D.; Qin, S.; Chen, Y. Porous boron nitride nanosheets for effective water cleaning. Nature Communications 2013, 4, 1777.

(8) Zhi, C.; Xu, Y.; Bando, Y.; Golberg, D. Highly thermo-conductive fluid with boron nitride nanofillers. ACS Nano 2011, 5, 6571-6577.

(9) Taha-Tijerina, J.; Narayanan, T. N.; Gao, G.; Rohde, M.; Tsentalovich, D. A.; Pasquali, M.; Ajayan, P. M. Electrically insulating thermal nano-oils using 2D fillers. ACS Nano 2012, 6, 1214-1220. 
(10) Krishnam, M.; Bose, S.; Das, C. Boron nitride $(\mathrm{BN})$ nanofluids as cooling agent in thermal management system (TMS). Applied Thermal Engineering 2016, 106, 951-958.

(11) Zhao, H.; Ding, J.; Xu, B.; Zhao, X.; Zheng, Y.; Shao, Z.; Yu, H. Green Synthesis of Graphene/Boron Nitride Composites for Ultrahigh Thermally Conductive Fluids. ACS Sustainable Chemistry and Engineering 2019, \%, 14266-14272.

(12) Ciofani, G.; Danti, S.; Genchi, G. G.; Mazzolai, B.; Mattoli, V. Boron nitride nanotubes: Biocompatibility and potential spill-over in nanomedicine. Small 2013, 9, 1672-1685.

(13) Weng, Q.; Wang, B.; Wang, X.; Hanagata, N.; Li, X.; Liu, D.; Wang, X.; Jiang, X.; Bando, Y.; Golberg, D. Highly water-soluble, porous, and biocompatible boron nitrides for anticancer drug delivery. ACS Nano 2014, 8, 6123-6130.

(14) Weng, Q.; Wang, X.; Wang, X.; Bando, Y.; Golberg, D. Functionalized hexagonal boron nitride nanomaterials: Emerging properties and applications. Chemical Society Reviews 2016, 45, 3989-4012.

(15) Secchi, E.; Marbach, S.; Niguès, A.; Stein, D.; Siria, A.; Bocquet, L. Massive radius-dependent flow slippage in carbon nanotubes. Nature 2016, 53\%, 210-213.

(16) Siria, A.; Poncharal, P.; Biance, A. L.; Fulcrand, R.; Blase, X.; Purcell, S. T.; Bocquet, L. Giant osmotic energy conversion measured in a single transmembrane boron nitride nanotube. Nature 2013, 494, 455-458.

(17) Grosjean, B.; Pean, C.; Siria, A.; Bocquet, L.; Vuilleumier, R.; Bocquet, M. L. Chemisorption of Hydroxide on 2D Materials from DFT Calculations: Graphene versus Hexagonal Boron Nitride. Journal of Physical Chemistry Letters 2016, 7, 4695-4700.
(18) Secchi, E.; Niguès, A.; Jubin, L.; Siria, A.; Bocquet, L. Scaling behavior for ionic transport and its fluctuations in individual carbon nanotubes. Physical Review Letters 2016, 116, 154501.

(19) Siria, A.; Bocquet, M.-L.; Bocquet, L. New avenues for the large-scale harvesting of blue energy. Nature Reviews Chemistry 2017, 1, 0091.

(20) Meyer, J. C.; Geim, A. K.; Katsnelson, M. I.; Novoselov, K. S.; Booth, T. J.; Roth, S. The structure of suspended graphene sheets. Nature 2007, 446, 6063.

(21) Fasolino, A.; Los, J. H.; Katsnelson, M. I. Intrinsic ripples in graphene. Nature $\mathrm{Ma}^{-}$ terials 2007, 6, 858-861.

(22) Guinea, F.; Horovitz, B.; Le Doussal, P. Gauge field induced by ripples in graphene. Physical Review B - Condensed Matter and Materials Physics 2008, 7r, 205421.

(23) Guinea, F.; Katsnelson, M. I.; Vozmediano, M. A. Midgap states and charge inhomogeneities in corrugated graphene. Physical Review B - Condensed Matter and Materials Physics 2008, 77, 075422.

(24) Vázquez De Parga, A. L.; Calleja, F.; Borca, B.; Passeggi, M. C.; Hinarejos, J. J.; Guinea, F.; Miranda, R. Periodically rippled graphene: Growth and spatially resolved electronic structure. Physical Review Letters 2008, 100, 056807.

(25) Boukhvalov, D. W.; Katsnelson, M. I. Enhancement of chemical activity in corrugated graphene. Journal of Physical Chemistry C 2009, 113, 14176-14178.

(26) Bao, W.; Miao, F.; Chen, Z.; Zhang, H.; Jang, W.; Dames, C.; Lau, C. N. Controlled ripple texturing of suspended graphene and ultrathin graphite membranes. Nature Nanotechnology 2009, 4, 562-566. 
(27) Ma, M.; Grey, F.; Shen, L.; Urbakh, M.; Wu, S.; Liu, J. Z.; Liu, Y.; Zheng, Q. Water transport inside carbon nanotubes mediated by phonon-induced oscillating friction. Nature Nanotechnology 2015, 10, 692-695.

(28) Ghoufi, A.; Szymczyk, A.; Malfreyt, P. Ultrafast diffusion of Ionic Liquids Confined in Carbon Nanotubes. Scientific Reports 2016, 6, 28518.

(29) Cruz-Chú, E. R.; Papadopoulou, E.; Walther, J. H.; Popadić, A.; Li, G.; Praprotnik, M.; Koumoutsakos, P. On phonons and water flow enhancement in carbon nanotubes. Nature Nanotechnology 2017, 12, 1106-1108.

(30) Marbach, S.; Dean, D. S.; Bocquet, L. Transport and dispersion across wiggling nanopores. Nature Physics 2018, 14, $1108-1113$

(31) Tersoff, J. New Empirical Model for the Structural Properties of Silicon. Physical Review Letters 1986, 56, 632-635.

(32) Tersoff, J. Empirical Interatomic Potential for Carbon, with Applications to Amorphous Carbon. Physical Review Letters 1988, 61, 2879-2882.

(33) Tersoff, J. Modeling solid-state chemistry: Interatomic potentials for multicomponent systems. Physical Review B 1989, 39, 5566-5568.

(34) Brenner, D. W. Empirical potential for hydrocarbons for use in simulating the chemical vapor deposition of diamond film. Physical Review B 1990, 42, 94589471.

(35) Albe, K.; Möller, W.; Heinig, K. H. Computer simulation and boron nitride. Radiation Effects and Defects in Solids 1997, 141, 85-97.

(36) Sekkal, W.; Bouhafs, B.; Aourag, H.; Certier, M. Molecular-dynamics simulation of structural and thermodynamic properties of boron nitride. Journal of Physics Condensed Matter 1998, 10, 4975-4984.

(37) Matsunaga, K.; Fisher, C.; Matsubara, H. Tersoff Potential Parameters for Simulating Cubic Boron Carbonitrides solid solution model Tersoff Potential Parameters for Simulating Cubic Boron Carbonitrides. Japanese Journal of Applied Physics 2000, 39, 48-51.

(38) Matsunaga, K.; Iwamoto, Y. Molecular Dynamics Study of Atomic Structure and Diffusion Behavior in Amorphous Silicon Nitride Containing Boron. Journal of the American Ceramic Society 2001, 84, 2213-2219.

(39) Sevik, C.; Kınacı, A.; Haskins, J. B.; Çağın, T. Characterization of thermal transport in low-dimensional boron nitride nanostructures. Physical Review B Condensed Matter and Materials Physics 2011, 84, 085409.

(40) Kinacı, A.; Haskins, J. B.; Sevik, C.; Çă̆ın, T. Thermal conductivity of BNC nanostructures. Physical Review B Condensed Matter and Materials Physics 2012, 86, 115410.

(41) Los, J. H.; Kroes, J. M.; Albe, K.; Gordillo, R. M.; Katsnelson, M. I.; Fasolino, A. Extended Tersoff potential for boron nitride: Energetics and elastic properties of pristine and defective h -BN. Physical Review B 2017, 96, 184108.

(42) van Duin, A. C. T.; Dasgupta, S.; Lorant, F.; Goddard III, W. A. ReaxFF: A Reactive Force Field for Hydrocarbons. Journal of Physical Chemistry A 2001, 105, 9396-9409.

(43) Chenoweth, K.; Van Duin, A. C.; Goddard, W. A. ReaxFF reactive force field for molecular dynamics simulations of hydrocarbon oxidation. Journal of Physical Chemistry A 2008, 112, 1040-1053. 
(44) Weismiller, M. R.; Duin, A. C.; Lee, J.; Yetter, R. A. ReaxFF reactive force field development and applications for molecular dynamics simulations of ammonia borane dehydrogenation and combustion. Journal of Physical Chemistry A 2010, 114, 5485-5492.

(45) Paupitz, R.; Junkermeier, C. E.; van Duin, A. C.; Branicio, P. S. Fullerenes generated from porous structures. Physical Chemistry Chemical Physics 2014, 16, 25515-25522.

(46) Liu, S.; Van Duin, A. C.; Van Duin, D. M.; Liu, B.; Edgar, J. H. Atomistic Insights into Nucleation and Formation of Hexagonal Boron Nitride on Nickel from First-Principles-Based Reactive Molecular Dynamics Simulations. ACS Nano 2017, 11, 3585-3596.

(47) Rajan, A. G.; Strano, M. S.; Blankschtein, D. Ab Initio Molecular Dynamics and Lattice Dynamics-Based Force Field for Modeling Hexagonal Boron Nitride in Mechanical and Interfacial Applications. Journal of Physical Chemistry Letters 2018, 9, 1584-1591.

(48) Mortazavi, B.; Rémond, Y. Investigation of tensile response and thermal conductivity of boron-nitride nanosheets using molecular dynamics simulations. Physica E: Low-Dimensional Systems and Nanostructures 2012, 44, 1846-1852.

(49) Liao, M.-L.; Lian, T.-W.; Ju, S.-P. Tensile and compressive behaviours of a boron nitride nanotube: Temperature effects. Materials Science Forum 2012, 700, 125-128.

(50) Krishnan, N. M. A.; Ghosh, D. Defect induced plasticity and failure mechanism of boron nitride nanotubes under tension. Journal of Applied Physics 2014, 116, 044313.

(51) Le, M. Q.; Nguyen, D. T. Atomistic simulations of pristine and defective hexagonal $\mathrm{BN}$ and $\mathrm{SiC}$ sheets under uniaxial tension. Materials Science and Engineering $A$ 2014, 615, 481-488.

(52) Hu, S.; Lozada-Hidalgo, M.; Wang, F. C.; Mishchenko, A.; Schedin, F.; Nair, R. R.; Hill, E. W.; Boukhvalov, D. W.; Katsnelson, M. I.; Dryfe, R. A. et al. Proton transport through one-atom-thick crystals. Nature 2014, 516, 227-230.

(53) Tocci, G.; Joly, L.; Michaelides, A. Friction of water on graphene and hexagonal boron nitride from $\mathrm{Ab}$ initio methods: Very different slippage despite very similar interface structures. Nano Letters 2014, 14, 6872-6877.

(54) Androulidakis, C.; Koukaras, E. N.; Poss, M.; Papagelis, K.; Galiotis, C.; Tawfick, S. Strained hexagonal boron nitride: Phonon shift and Grüneisen parameter. Physical Review B 2018, 9\%, 241414.

(55) Simonnin, P.; Noetinger, B.; NietoDraghi, C.; Marry, V.; Rotenberg, B. Diffusion under Confinement: Hydrodynamic Finite-Size Effects in Simulation. Journal of Chemical Theory and Computation 2017, 13, 2881-2889.

(56) Los, J. H.; Katsnelson, M. I.; Yazyev, O. V.; Zakharchenko, K. V.; Fasolino, A. Scaling properties of flexible membranes from atomistic simulations: Application to graphene. Physical Review B - Condensed Matter and Materials Physics 2009, 80, 1-4.

(57) Rupp, M.; Tkatchenko, A.; Müller, K. R.; Von Lilienfeld, O. A. Fast and accurate modeling of molecular atomization energies with machine learning. Physical Review Letters 2012, 108, 058301.

(58) Behler, J. Perspective : Machine learning potentials for atomistic simulations. Journal of Chemical Physics 2016, 145, 170901. 
(59) Bartók, A. P.; Csányi, G.; Kermode, J. R.; De, S.; Bernstein, N.; Ceriotti, M.; Bartók, A. P.; Poelking, C. Machine learning unifies the modeling of materials and molecules. Science Advances 2017, 3, e1701816.

(60) Botu, V.; Batra, R.; Chapman, J.; Ramprasad, R. Machine learning force fields: Construction, validation, and outlook. Journal of Physical Chemistry C 2017, 121, 511-522.

(61) Li, Z.; Wang, S.; Chin, W. S.; Achenie, L. E.; Xin, H. High-throughput screening of bimetallic catalysts enabled by machine learning. Journal of Materials Chemistry A 2017, 5, 24131-24138.

(62) Kitchin, J. R. Machine learning in catalysis. Nature Catalysis 2018, 1, 230-232.

(63) Goldsmith, B. R.; Esterhuizen, J.; Liu, J. X.; Bartel, C. J.; Sutton, C. Machine learning for heterogeneous catalyst design and discovery. AIChE Journal 2018, 64, 2311-2323.

(64) Behler, J.; Parrinello, M. Generalized neural-network representation of high-dimensional potential-energy surfaces. Physical Review Letters 2007, 98, 146401.

(65) Bartók, A. P.; Payne, M. C.; Kondor, R.; Csányi, G. Gaussian Approximation Potentials : The Accuracy of Quantum Mechanics, without the Electrons. Physical Review Letters 2010, 104, 136403.

(66) Li, Z.; Kermode, J. R.; De Vita, A. Molecular Dynamics with On-theFly Machine Learning of QuantumMechanical Forces. Physical Review Letters 2015, 114, 096405.

(67) Chmiela, S.; Tkatchenko, A.; Sauceda, H. E.; Poltavsky, I.; Schütt, K. T.; Müller, K. R. Machine learning of accurate energy-conserving molecular force fields. Science Advances 2017, 3, e1603015.
(68) Nguyen, T. T.; Székely, E.; Imbalzano, G.; Behler, J.; Csányi, G.; Ceriotti, M.; Götz, A. W.; Paesani, F. Comparison of permutationally invariant polynomials, neural networks, and Gaussian approximation potentials in representing water interactions through manybody expansions. Journal of Chemical Physics 2018, 148, 241725.

(69) Bartók, A. P.; Csányi, G. Gaussian approximation potentials: A brief tutorial introduction. International Journal of Quantum Chemistry 2015, 115, 10511057.

(70) Rowe, P.; Csányi, G.; Alfè, D.; Michaelides, A. Development of a machine learning potential for graphene. Physical Review B 2018, 97, 054303.

(71) Deringer, V. L.; Csányi, G. Machine learning based interatomic potential for amorphous carbon. Physical Review B 2017, 95, 094203.

(72) Rowe, P.; Deringer, V. L.; Gasparotto, P.; Csányi, G.; Michaelides, A. An accurate and transferable machine learning potential for carbon. Journal of Chemical Physics 2020, 153.

(73) Szlachta, W. J.; Bartók, A. P.; Csányi, G. Accuracy and transferability of GAP models for tungsten. Physical Review $B$ 2014, 90, 104108.

(74) Jinnouchi, R.; Lahnsteiner, J.; Karsai, F.; Kresse, G.; Bokdam, M. Phase Transitions of Hybrid Perovskites Simulated by Machine-Learning Force Fields Trained on the Fly with Bayesian Inference. Physical Review Letters 2019, 122, 225701.

(75) De, S.; Bartók, A. P.; Csányi, G.; Ceriotti, M. Comparing molecules and solids across structural and alchemical space. Physical Chemistry Chemical Physics 2016, 18, 13754-13769. 
(76) Bartók, A. P.; Kondor, R.; Csányi, G. On representing chemical environments. Physical Review B - Condensed Matter and Materials Physics 2013, 87, 184115.

(77) Smith, J. S.; Nebgen, B.; Lubbers, N.; Isayev, O.; Roitberg, A. E. Less is more: Sampling chemical space with active learning. Journal of Chemical Physics 2018, 148 .

(78) Ceriotti, M.; Tribello, G. A.; Parrinello, M. Simplifying the representation of complex free-energy landscapes using sketch-map. Proceedings of the National Academy of Sciences 2011, 108, 1302313028 .

(79) Tribello, G. A.; Ceriotti, M.; Parrinello, M. Using sketch-map coordinates to analyze and bias molecular dynamics simulations. Proceedings of the National Academy of Sciences of the United States of America 2012, 109, 5196-5201.

(80) Kresse, G.; Hafner, J. Ab initio molecular dynamics for liquid metals. Physical Review B 1993, 47, 558-561.

(81) Kresse, G.; Hafner, J. Ab initio molecular-dynamics simulation of the liquid-metalamorphous- semiconductor transition in germanium. Physical Review B 1994, 49, 14251-14269.

(82) Kresse, G.; Furthmüller, J. Efficient iterative schemes for ab initio total-energy calculations using a plane-wave basis set. Physical Review B - Condensed Matter and Materials Physics 1996, 54, 11169 11186.

(83) Kresse, G.; Furthmüller, J. Efficiency of ab-initio total energy calculations for metals and semiconductors using a planewave basis set. Computational Materials Science 1996, 6, 15-50.

(84) Perdew, J. P.; Burke, K.; Ernzerhof, M. Generalized gradient approximation made simple. Physical Review Letters 1996, 77, 3865-3868.
(85) Grimme, S.; Antony, J.; Ehrlich, S.; Krieg, H. A consistent and accurate ab initio parametrization of density functional dispersion correction (DFT-D) for the 94 elements H-Pu. Journal of Chemical Physics 2010, 132.

(86) Grimme, S.; Ehrlich, S.; Goerigk, L. Effect of the Damping Function in Dispersion Corrected Density Functional Theory. Journal of Computational Chemistry 2011, 32, 1456.

(87) Blöchl, P. E. Projector augmented-wave method. Physical Review B 1994, 50, 17953-17979.

(88) Kresse, G.; Joubert, D. From ultrasoft pseudopotentials to the projector augmented-wave method. Physical Review B - Condensed Matter and Materials Physics 1999, 59, 1758-1775.

(89) Plimpton, S. Fast Parallel Algorithms for Short-Range Molecular Dynamics. Journal of Computational Physics 1995, 117, $1-19$.

(90) Leven, I.; Azuri, I.; Kronik, L.; Hod, O. Inter-layer potential for hexagonal boron nitride. Journal of Chemical Physics 2014, 140, 104106.

(91) Leven, I.; Maaravi, T.; Azuri, I.; Kronik, L.; Hod, O. Interlayer Potential for Graphene/h-BN Heterostructures. Journal of Chemical Theory and Computation 2016, 12, 2896-2905.

(92) Maaravi, T.; Leven, I.; Azuri, I.; Kronik, L.; Hod, O. Interlayer Potential for Homogeneous Graphene and Hexagonal Boron Nitride Systems: Reparametrization for Many-Body Dispersion Effects. Journal of Physical Chemistry C 2017, 121, 22826-22835.

(93) Solozhenko, V. L.; Will, G.; Elf, F. Isothermal compression of hexagonal graphite-like boron nitride up to $12 \mathrm{GPa}$. Solid State Communications 1995, 96, $1-3$. 
(94) Cerda, E.; Mahadevan, L. Geometry and Physics of Wrinkling. Physical Review Letters 2003, 90, 074302.

(95) Bosak, A.; Serrano, J.; Krisch, M.; Watanabe, K.; Taniguchi, T.; Kanda, H. Elasticity of hexagonal boron nitride: Inelastic x-ray scattering measurements. Physical Review B - Condensed Matter and Materials Physics 2006, 73, 041402(R).

(96) Graziano, G.; Klimeš, J.; FernandezAlonso, F.; Michaelides, A. Improved description of soft layered materials with van der Waals density functional theory. Journal of Physics: Condensed Matter 2012, 24, 424216.

(97) Ohba, N.; Miwa, K.; Nagasako, N.; Fukumoto, A. First-principles study on structural, dielectric, and dynamical properties for three BN polytypes. Physical Review $B$ - Condensed Matter and Materials Physics 2001, 63, 115207.

(98) Murnaghan, F. D. The Compressibility of Media Under Extreme Pressure. Proceedings of the National Academy of Sciences of the United States of America 1944, 30, 244-247.

(99) Ma, M.; Tocci, G.; Michaelides, A.; Aeppli, G. Fast diffusion of water nanodroplets on graphene. Nature Materials 2016, 15, 66-71.

(100) Alfè, D. PHON: A program to calculate phonons using the small displacement method. Computer Physics Communications 2009, 180, 2622-2633.

(101) Giannozzi, P.; de Gironcoli, S.; Pavone, P.; Baroni, S. Ab initio calculation of phonon dispersions in semiconductors. Physical Review $B$ 1991, 43, 7231-7242.

(102) Gonze, X.; Charlier, J. C.; Allan, D. C.; Teter, M. P. Interatomic force constants from first principles: The case of $\alpha$ quartz. Physical Review B 1994, 50, 13035-13038.

(103) Gonze, X.; Lee, C. Dynamical matrices, Born effective charges, dielectric permittivity tensors, and interatomic force constants from density-functional perturbation theory. Physical Review B Condensed Matter and Materials Physics 1997, 55, 10355-10368.

(104) Baroni, S.; de Gironcoli, S.; Dal Corso, A. Phonons and related crystal properties from density-functional perturbation theory. Reviews of Modern Physics 2001, 73, $515-557$.

(105) Sohier, T.; Gibertini, M.; Calandra, M.; Mauri, F.; Marzari, N. Breakdown of Optical Phonons' Splitting in TwoDimensional Materials. Nano Letters 2017, 17, 3758-3763.

(106) Giannozzi, P.; Baroni, S.; Bonini, N.; Calandra, M.; Car, R.; Cavazzoni, C.; Ceresoli, D.; Chiarotti, G. L.; Cococcioni, M.; Dabo, I. et al. QUANTUM ESPRESSO: A modular and open-source software project for quantum simulations of materials. Journal of Physics Condensed Matter 2009, 21, 395502.

(107) Giannozzi, P.; Andreussi, O.; Brumme, T.; Bunau, O.; Buongiorno Nardelli, M.; Calandra, M.; Car, R.; Cavazzoni, C.; Ceresoli, D.; Cococcioni, M. et al. Advanced capabilities for materials modelling with Quantum ESPRESSO. Journal of Physics Condensed Matter 2017, 29, 465901.

(108) Togo, A.; Tanaka, I. First principles phonon calculations in materials science. Scripta Materialia 2015, 108, 1-5.

(109) Geick, R.; Perry, C. H.; Rupprecht, G. Normal modes in hexagonal boron nitride. Physical Review 1966, 146, 543547. 
(110) Nemanich, R. J.; Solin, S. A.; Martin, R. M. Light scattering study of boron nitride microcrystals. Physical Review $B$ 1981, 23, 6348-6356.

(111) Reich, S.; Ferrari, A. C.; Arenal, R.; Loiseau, A.; Bello, I.; Robertson, J. Resonant Raman scattering in cubic and hexagonal boron nitride. Physical Review $B$ - Condensed Matter and Materials Physics 2005, 71, 205201.

(112) Serrano, J.; Bosak, A.; Arenal, R.; Krisch, M.; Watanabe, K.; Taniguchi, T.; Kanda, H.; Rubio, A.; Wirtz, L. Vibrational Properties of Hexagonal Boron Nitride: Inelastic X-Ray Scattering and Ab Initio Calculations. Physical Review Letters 2007, 98, 095503.

(113) Wirtz, L.; Rubio, A.; De La Concha, R.; Loiseau, A. Ab initio calculations of the lattice dynamics of boron nitride nanotubes. Physical Review B - Condensed Matter and Materials Physics 2003, 68, 045425 .

(114) Zakharchenko, K. V.; Los, J. H.; Katsnelson, M. I.; Fasolino, A. Atomistic simulations of structural and thermodynamic properties of bilayer graphene. Physical Review B - Condensed Matter and Materials Physics 2010, 81, 235439.

(115) Slotman, G. J.; Fasolino, A. Structure, stability and defects of single layer hexagonal BN in comparison to graphene. Journal of Physics Condensed Matter 2013, 25, 045009.

(116) Katsnelson, M. I.; Fasolino, A. Graphene as a prototype crystalline membrane. Accounts of Chemical Research 2013, 46, 97-105.

(117) Xu, P.; Neek-Amal, M.; Barber, S. D.; Schoelz, J. K.; Ackerman, M. L.; Thibado, P. M.; Sadeghi, A.; Peeters, F. M. Unusual ultra-lowfrequency fluctuations in freestanding graphene. Nature Communications 2014, 5, 3720.
(118) Nelson, D. R.; Peliti, L. Fluctuations in Membranes With Crystalline and Hexatic Order. Journal de physique Paris 1987, 48, 1085-1092.

(119) Le Doussal, P.; Radzihovsky, L. Selfconsistent theory of polymerized membranes. Physical Review Letters 1992, 69, 1209-1212.

(120) Nelson, D. R., Piran, T., Weinberg, S., Eds. Statistical Mechanics of Membranes and Surfaces, 2nd ed.; World Scientific: Singapore, 2004.

(121) Kownacki, J. P.; Mouhanna, D. Crumpling transition and flat phase of polymerized phantom membranes. Physical Review E - Statistical, Nonlinear, and Soft Matter Physics 2009, 79, 040101(R).

(122) Karssemeijer, L. J.; Fasolino, A. Phonons of graphene and graphitic materials derived from the empirical potential LCBOPII. Surface Science 2011, 605, 1611-1615.

(123) Wu, J.; Wang, B.; Wei, Y.; Yang, R.; Dresselhaus, M. Mechanics and mechanically tunable band gap in single-layer hexagonal boron-nitride. Materials Research Letters 2013, 1, 200-206.

(124) Wei, Y.; Wang, B.; Wu, J.; Yang, R.; Dunn, M. L. Bending rigidity and Gaussian bending stiffness of single-layered graphene. Nano Letters 2013, 13, 26-30.

(125) Zakharchenko, K. V.; Katsnelson, M. I.; Fasolino, A. Finite temperature lattice properties of graphene beyond the quasiharmonic approximation. Physical Review Letters 2009, 102, 046808.

(126) Thomas, S.; Ajith, K. M.; Valsakumar, M. C. Effect of ripples on the finite temperature elastic properties of hexagonal boron nitride using strain-fluctuation method. Superlattices and Microstructures 2017, 111, 360-372. 
(127) De Lima, A. L.; Müssnich, L. A.; Manhabosco, T. M.; Chacham, H.; Batista, R. J.; De Oliveira, A. B. Soliton instability and fold formation in laterally compressed graphene. Nanotechnology 2015, 26, 045707.

(128) Shankar, R.; Marchesini, S.; Petit, C. Enhanced Hydrolytic Stability of Porous Boron Nitride via the Control of Crystallinity, Porosity, and Chemical Composition. Journal of Physical Chemistry C 2019, 123, 4282-4290.

(129) Kroes, J. M.; Fasolino, A.; Katsnelson, M. I. Density functional based simulations of proton permeation of graphene and hexagonal boron nitride. Physical Chemistry Chemical Physics 2017, 19, 5813-5817.

(130) Liang, T.; He, G.; Wu, X.; Ren, J.; Guo, H.; Kong, Y.; Iwai, H.; Fujita, D.; Gao, H.; Guo, H. et al. Permeation through graphene ripples. 2D Materials 2017, 4, 025010.

(131) Sun, P. Z.; Yang, Q.; Kuang, W. J.; Stebunov, Y. V.; Xiong, W. Q.; Yu, J.; Nair, R. R.; Katsnelson, M. I.; Yuan, S. J.; Grigorieva, I. V. et al. Limits on gas impermeability of graphene. $\mathrm{Na}$ ture 2020, 579, 229-232. 
Graphical TOC Entry

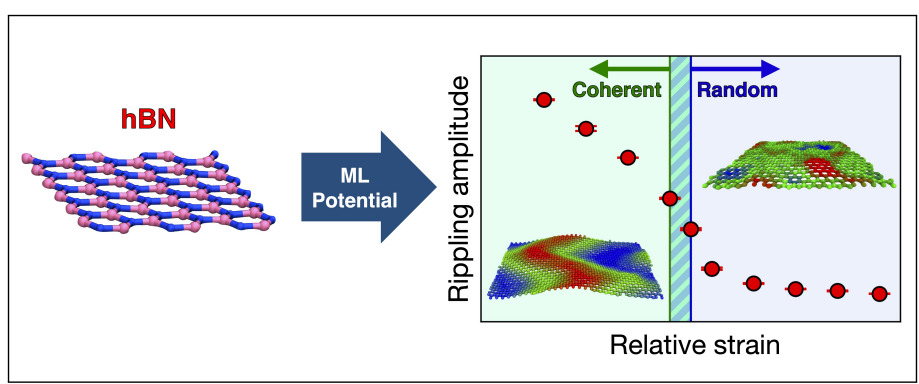

